\title{
Global solution of nematic liquid crystals models
}

\author{
Francisco GUILLÉN-GONZÁLEZ a , Marko ROJAS-MEDAR b
}

\begin{abstract}
We prove existence of a global weak solution for a nematic liquid crystal problem by means of a penalization method using a simplified Ericksen-Leslie model and a new compactness property for the gradient of the director field.
\end{abstract}

\section{Introduction}

In this Note, we establish the well-posedness in the large of a nematic liquid crystals model (formulated for instance in [7]) by means of a penalisation argument using a simplified Ericksen-Leslie model with the Ginzburg-Landau approximation $[1,2]$.

Let us consider a simplified version of the Ericksen-Leslie model, introduced by Lin in [4] and analysed by Lin and Liu [5, 6] who used a modified Galerkin approach, and by Shkoller [8] who relied on a contraction mapping argument coupled with appropriate energy estimates. This model is a modified Navier-Stokes system that take into account of the liquid crystallinity, coupling with the GinzburgLandau equations. A full version of this Ericksen-Leslie model has been recently studied by Coutand and Shkoller in [3], where local well-posedness (global for small enough data) is proved. Now, we are interested in the asymptotic behaviour respect to the penalisation parameter.

The unknowns are the time-dependent divergence-free velocity field $\mathbf{u}(t, \mathbf{x})$ and pressure $p(t, \mathbf{x})$ of the fluid and the director field $\mathbf{d}(t, \mathbf{x})$ representing the orientation of the liquid crystals molecules. The fluid is confined in an open bounded domain $\Omega \subset \mathbb{R}^{n}\left(n=2\right.$ or 3 ) with boundary $\partial \Omega$ of $C^{2}$ type.

In the penalised model one verifies the constraint $|\mathbf{d}| \leq 1$ as consequence of a maximum principle for the Ginzburg-Landau equation where the approximation

$$
\mathbf{f}_{\varepsilon}(\mathbf{d})=\varepsilon^{-2}\left(|\mathbf{d}|^{2}-1\right) \mathbf{d}
$$

is considered $(\varepsilon>0)$. Here $|\mathbf{d}|=|\mathbf{d}(t, \mathbf{x})|$ denotes the punctual Euclidean norm in $\mathbb{R}^{n}$. This penalisation function exhibits potential structure, i.e. there exists a potential function

$$
F_{\varepsilon}(\mathbf{d})=\varepsilon^{-2}\left(|\mathbf{d}|^{2}-1\right)^{2}
$$

such that $f_{\varepsilon}(\mathbf{d})=\nabla_{\mathbf{d}}\left(F_{\varepsilon}(\mathbf{d})\right)$ for all $\mathbf{d} \in \mathbb{R}^{n}$.

Accordingly, we consider the penalised model in $(0, T) \times \Omega$ as follows

$$
\begin{aligned}
& |\mathbf{d}| \leq 1, \quad \partial_{t} \mathbf{d}+\mathbf{u} \cdot \nabla \mathbf{d}+\gamma\left(\mathbf{f}_{\varepsilon}(\mathbf{d})-\Delta \mathbf{d}\right)=0 \\
& \partial_{t} \mathbf{u}+\mathbf{u} \cdot \nabla \mathbf{u}-\nu \Delta \mathbf{u}+\nabla p+\lambda \nabla \cdot(\nabla \mathbf{d} \odot \nabla \mathbf{d})=0 \\
& \nabla \cdot \mathbf{u}=0 \\
& \mathbf{u}_{\mid \partial \Omega}=0, \quad \mathbf{d}_{\mid \partial \Omega}=\mathbf{h} \\
& \mathbf{u}_{\mid t=0}=\mathbf{u}_{0}, \quad \mathbf{d}_{\mid t=0}=\mathbf{d}_{0}
\end{aligned}
$$


Here, $\mathbf{u}_{0}$ and $\mathbf{d}_{0}$ are, respectively, the initial velocity and director fields. In order to obtain the dissipativity of the model, we shall assume (as in all previous works) time independent (Dirichlet) boundary data for the director field $\mathbf{d}$, given by $\mathbf{h}: \partial \Omega \rightarrow \mathbb{R}^{n}$. Concerning the coefficients, $\nu>0$ represents the viscosity of the fluid, $\lambda>0$ is an elasticity constant, $\varepsilon>0$ is a penalisation parameter (with respect to the unitary constraint), and $\gamma>0$ is a relaxation-time constant. We have used the tensorial notation

$$
(\nabla \mathbf{d} \odot \nabla \mathbf{d})_{i j}=\sum_{k=1}^{n} \partial_{x_{i}} d_{k} \partial_{x_{j}} d_{k}
$$

In the $\varepsilon$-limit model we will find the restriction $|\mathbf{d}|=1$ and the Lagrange multiplier associated $|\nabla \mathbf{d}|^{2} \mathbf{d}$. Indeed, when $\varepsilon \rightarrow 0$ we will find a limit problem, where it changes (1) by

$$
|\mathbf{d}|=1, \quad \partial_{t} \mathbf{d}+\mathbf{u} \cdot \nabla \mathbf{d}-\gamma \Delta \mathbf{d}-\gamma|\nabla \mathbf{d}|^{2} \mathbf{d}=0 .
$$

Let us introduce the following space of functions

$$
\begin{aligned}
\mathbf{H}_{\mathbf{h}}^{1} & =\left\{\mathbf{d} \in H^{1}(\Omega)^{n} / \mathbf{d}=\mathbf{h} \text { on } \partial \Omega\right\} \\
\mathbf{H} & =\left\{\mathbf{u} \in L^{2}(\Omega)^{n} / \nabla \cdot \mathbf{u}=0, \mathbf{u} \cdot \mathbf{n}=0 \text { on } \partial \Omega\right\} \\
\mathbf{V} & =\left\{\mathbf{u} \in H_{0}^{1}(\Omega)^{n} / \nabla \cdot \mathbf{u}=0\right\} .
\end{aligned}
$$

For simplicity, let us denote $\mathbf{L}^{2}, \mathbf{H}^{1}$ instead of $L^{2}(\Omega)^{n}, H^{1}(\Omega)^{n}$ etc. Our main result is the following

Theorem 1.1 Let $T>0$ and $\Omega \subset \mathbb{R}^{n}$ be an open, bounded and $C^{2}$ domain. Let us assume $\mathbf{u}_{0} \in \mathbf{H}$, $\mathbf{d}_{0} \in \mathbf{H}_{\mathbf{h}}^{1}$ and $\mathbf{h} \in \mathbf{H}^{2}$ such that $\left|\mathbf{d}_{0}\right|=1$ in $\Omega$ and $|\mathbf{h}|=1$ on $\partial \Omega$. Then, there exists a global weak solution $\mathbf{u} \in L^{2}(0, T ; \mathbf{V}) \cap L^{\infty}(0, T ; \mathbf{H}), \mathbf{d} \in L^{\infty}\left(0, T ; \mathbf{H}_{\mathbf{h}}^{1}\right)$ of the limit problem (2)-(6) obtained as a limit of "semi-strong" solutions $\mathbf{u}_{\varepsilon} \in L^{2}(0, T ; \mathbf{V}) \cap L^{\infty}(0, T ; \mathbf{H}), \mathbf{d}_{\varepsilon} \in L^{2}\left(0, T ; \mathbf{H}^{2} \cap \mathbf{H}_{\mathbf{h}}^{1}\right) \cap L^{\infty}\left(0, T ; \mathbf{H}_{\mathbf{h}}^{1}\right)$ of the coupled Navier-Stokes and Ginzburg-Landau model (1)-(5) as $\varepsilon$ goes to zero.

Remark 1 Up to our known, this theorem is the first result of existence of a global in time solution (without restrictions on the data) of the limit problem (2)-(6). In [7], Prohl proves existence (and uniqueness) of a local in time strong solution of (2)-(6). On the other hand, Lin and Liu studied in [6] the asymptotic behaviour of (1)-(5) when $\varepsilon$ goes to zero, but the limit of the (strongly) nonlinear terms $\nabla \mathbf{d}_{\varepsilon} \odot \nabla \mathbf{d}_{\varepsilon}$ is only obtained towards a measure valued tensor $M$. The main contribution in this note is to identify $M$ with the limit tensor $\nabla \mathbf{d} \odot \nabla \mathbf{d}$.

Remark 2 Notice that in the $\varepsilon$-limit problem (2)-(6) one loses the $H^{2}$-regularity for the director field $\mathbf{d}$, hence although $\left(\mathbf{u}_{\varepsilon}, \mathbf{d}_{\varepsilon}\right)$ verifies (1) point-wise a.e. $(t, \mathbf{x})$, their limit $(\mathbf{u}, \mathbf{d})$ verifies (6) only in a distributional sense.

\section{$2 \quad \varepsilon$-approximate solutions and dissipativity}

For each $\varepsilon>0$, let us consider a "semi-strong" solution $\left(\mathbf{u}_{\varepsilon}, \mathbf{d}_{\varepsilon}\right)$ of the $\varepsilon$-approximate problem (1)-(5), that is

$$
\mathbf{u}_{\varepsilon} \in L^{2}(0, T ; \mathbf{V}) \cap L^{\infty}(0, T ; \mathbf{H}), \quad \mathbf{d}_{\varepsilon} \in L^{2}\left(0, T ; \mathbf{H}^{2} \cap \mathbf{H}_{\mathbf{h}}^{1}\right) \cap L^{\infty}\left(0, T ; \mathbf{H}_{\mathbf{h}}^{1}\right),
$$


verifying the $\mathbf{u}$-system (2) in the distributional sense and the $\mathbf{d}$-system (1) point-wise a.e. The boundary conditions are verified in the trace sense. Finally, the initial conditions have classical sense; indeed $\mathbf{u}_{\varepsilon}$ and $\mathbf{d}_{\varepsilon}$ are time-continuous functions, as consequence of the additional regularity

$$
\partial_{t} \mathbf{u}_{\varepsilon} \in L^{p}\left(0, T ; \mathbf{V}^{\prime}\right) \quad \text { and } \quad \partial_{t} \mathbf{d}_{\varepsilon} \in L^{p}\left(0, T ; \mathbf{L}^{2}\right)(p=2 \text { if } n=2 \text { or } p=4 / 3 \text { if } n=3)
$$

that can be obtained applying the previous regularity (7) to the equations (1) and (2).

The existence of $\left(\mathbf{u}_{\varepsilon}, \mathbf{d}_{\varepsilon}\right)$ can be proved ([5]) by means of three main arguments: a semi-galerkin method (space-discretization of the $\mathbf{u}$-system (2), remaining the $\mathbf{d}$-system (1) in the continuous sense), a maximum principle for the $\mathbf{d}$-system in order to obtain the constraint $\left|\mathbf{d}_{\varepsilon}\right| \leq 1$ and the following energy inequality,

$$
\frac{d}{d t}\left(\frac{1}{2}\left\|\mathbf{u}_{\varepsilon}\right\|^{2}+\frac{\lambda}{2}\left\|\nabla \mathbf{d}_{\varepsilon}\right\|^{2}+\lambda \int_{\Omega} F_{\varepsilon}\left(\mathbf{d}_{\varepsilon}\right) d \mathbf{x}\right)+\nu\left\|\nabla \mathbf{u}_{\varepsilon}\right\|^{2}+\lambda \gamma\left\|\mathbf{f}_{\varepsilon}\left(\mathbf{d}_{\varepsilon}\right)-\Delta \mathbf{d}_{\varepsilon}\right\|^{2} \leq 0,
$$

obtained taking respectively $\lambda\left(\mathbf{f}_{\varepsilon}\left(\mathbf{d}_{\varepsilon}\right)-\Delta \mathbf{d}_{\varepsilon}\right)$ and $\mathbf{u}_{\varepsilon}$ as test functions in (1)-(2) and using the equality $\nabla \cdot(\nabla \mathbf{d} \odot \nabla \mathbf{d})=\nabla\left(|\nabla \mathbf{d}|^{2} / 2\right)+\nabla \mathbf{d}^{t} \Delta \mathbf{d}$. In $(8),\|\cdot\|$ denotes the $L^{2}(\Omega)$-norm.

\section{$3 \quad \varepsilon$-independent estimates}

For simplicity, let us denote $L^{2}\left(\mathbf{H}^{1}\right)$ instead of $L^{2}\left(0, T ; \mathbf{H}^{1}\right)$, etc. From the maximum principle in (2)

$$
\left|\mathbf{d}_{\varepsilon}\right| \leq 1 \quad \text { a.e. }(t, \mathbf{x}) \text {. }
$$

On the other hand, from the energy inequality (8), one has the following ( $\varepsilon$-independent) estimates:

$$
\begin{array}{rll}
\mathbf{u}_{\varepsilon} & \text { is bounded in } & L^{\infty}(\mathbf{H}) \cap L^{2}(\mathbf{V}), \\
\mathbf{d}_{\varepsilon} & \text { is bounded in } & L^{\infty}\left(0, T ; \mathbf{H}_{\mathbf{h}}^{1}\right), \\
\mathbf{w}_{\varepsilon}:=\mathbf{f}_{\varepsilon}\left(\mathbf{d}_{\varepsilon}\right)-\Delta \mathbf{d}_{\varepsilon} & \text { is bounded in } & L^{2}\left(\mathbf{L}^{2}\right), \\
F_{\varepsilon}\left(\mathbf{d}_{\varepsilon}\right) & \text { is bounded in } & L^{\infty}\left(L^{1}\right) .
\end{array}
$$

Moreover, applying estimates (9)-(11) in equations (1)-(2),

$$
\begin{array}{lll}
\partial_{t} \mathbf{u}_{\varepsilon} & \text { is bounded in } & L^{2}\left(\mathbf{V}^{\prime}\right)+L^{\infty}\left(\left(\mathbf{W}^{1, r} \cap \mathbf{V}\right)^{\prime}\right), r>n, \\
\partial_{t} \mathbf{d}_{\varepsilon} \quad \text { is bounded in } & L^{2}\left(\mathbf{L}^{2}\right)+L^{q}\left(\mathbf{L}^{4 / 3}\right), q=4 \text { if } n=2 \text { or } q=8 / 3 \text { if } n=3 .
\end{array}
$$

Finally, (10) implies in particular

$$
M_{\varepsilon}:=\nabla \mathbf{d}_{\varepsilon} \odot \nabla \mathbf{d}_{\varepsilon} \quad \text { is bounded in } \quad L^{\infty}\left(\mathbf{L}^{1}\right) .
$$




\section{$4 \varepsilon$-convergence}

From previous estimates (9)-(15) and compactness results of Aubin-Lions type [9], there exists subsequences (equally denoted) $\mathbf{d}_{\varepsilon}, \mathbf{u}_{\varepsilon}, \mathbf{w}_{\varepsilon}, M_{\varepsilon}$ and their respective limit functions $\mathbf{d}, \mathbf{u}, \mathbf{w}, M$, such that

$$
\begin{array}{rll}
\mathbf{u}_{\varepsilon} \rightarrow \mathbf{u} & \text { in } & L^{\infty}(\mathbf{H}) \text { weak } \star, L^{2}(\mathbf{V}) \text { weak, } L^{2}(\mathbf{H}) \text { strong } \\
\mathbf{d}_{\varepsilon} \rightarrow \mathbf{d} & \text { in } & L^{\infty}\left(\mathbf{H}^{1}\right) \text { weak } \star C\left(\mathbf{L}^{2}\right) \text { strong, } \\
\partial_{t} \mathbf{u}_{\varepsilon} \rightarrow \partial_{t} \mathbf{u} & \text { in } & L^{2}\left(\mathbf{V}^{\prime}\right)+L^{\infty}\left(\left(\mathbf{W}^{1, r} \cap \mathbf{V}\right)^{\prime}\right) \text { weak, } \\
\partial_{t} \mathbf{d}_{\varepsilon} \rightarrow \partial_{t} \mathbf{d} & \text { in } & L^{2}\left(\mathbf{L}^{2}\right)+L^{p}\left(\mathbf{L}^{4 / 3}\right) \text { weak } \\
\mathbf{w}_{\varepsilon} \rightarrow \mathbf{w} & \text { in } & L^{2}\left(\mathbf{L}^{2}\right) \text { weak, } \\
\nabla \mathbf{d}_{\varepsilon} \odot \nabla \mathbf{d}_{\varepsilon} \rightarrow M & \text { in the measure sense. }
\end{array}
$$

Consequently, taking limits as $\varepsilon \rightarrow 0$ and using De Rham's lemma [10], we arrive at

$$
\begin{aligned}
& \partial_{t} \mathbf{d}+\mathbf{u} \cdot \nabla \mathbf{d}+\gamma \mathbf{w}=0 \\
& \partial_{t} \mathbf{u}+\mathbf{u} \cdot \nabla \mathbf{u}-\nu \Delta \mathbf{u}+\nabla p+\lambda \nabla \cdot M=0 \\
& \nabla \cdot \mathbf{u}=0 \\
& \mathbf{u}_{\mid \partial \Omega}=0, \quad \mathbf{d}_{\mid \partial \Omega}=\mathbf{h} \\
& \mathbf{u}_{\mid t=0}=\mathbf{u}_{0}, \quad \mathbf{d}_{\mid t=0}=\mathbf{d}_{0}
\end{aligned}
$$

On the other hand, since $\varepsilon^{-1}\left(\left|\mathbf{d}_{\varepsilon}\right|^{2}-1\right.$ ) is bounded in $L^{\infty}\left(L^{2}\right)$ (using (12)) and $\mathbf{d}_{\varepsilon} \rightarrow \mathbf{d}$ point-wise a.e. $(t, \mathbf{x})$, one get the unity constraint $|\mathbf{d}(t, \mathbf{x})|=1$ a.e. $(t, \mathbf{x})$. Therefore, in order to finish the proof of Theorem 1.1, we have to identify w with $-\Delta \mathbf{d}-|\nabla \mathbf{d}|^{2} \mathbf{d}$ and $M$ with $\nabla \mathbf{d} \odot \nabla \mathbf{d}$.

\section{$5 \quad$ Identification of $\mathbf{w}=-\Delta \mathbf{d}-|\nabla \mathbf{d}|^{2} \mathbf{d}$.}

The argument of this section is based in the known literature on harmonic functions with values in the unit sferic surface (see for instance [1, 2] and references therein cited). In particular, we will use the following result, which is a slightly modification (introducing the convection terms) of Lemma 2.2 in [2] (see also Lemma 7.1 in $[6]$ ):

Lemma 5.1 The following two systems are equivalent:

$$
\partial_{t} \mathbf{d}+\mathbf{u} \cdot \nabla \mathbf{d}-\gamma \Delta \mathbf{d}=\gamma|\nabla \mathbf{d}|^{2} \mathbf{d}
$$

and

$$
|\mathbf{d}|=1, \quad\left(\partial_{t} \mathbf{d}+\mathbf{u} \cdot \nabla \mathbf{d}\right) \wedge \mathbf{d}-\gamma \nabla \cdot(\nabla \mathbf{d} \wedge \mathbf{d})=0 .
$$

Since we already have that $|\mathbf{d}|=1$, it suffices to verify the equation of (21). Indeed, making the vectorial product of equation (2) by $\mathbf{d}_{\varepsilon}$, taking into account that $\mathbf{f}_{\varepsilon}\left(\mathbf{d}_{\varepsilon}\right) \wedge \mathbf{d}_{\varepsilon}=0$ and $-\Delta \mathbf{d}_{\varepsilon} \wedge \mathbf{d}_{\varepsilon}=-\nabla \cdot\left(\nabla \mathbf{d}_{\varepsilon} \wedge \mathbf{d}_{\varepsilon}\right)$, one has

$$
\left(\partial_{t} \mathbf{d}_{\varepsilon}+\mathbf{u}_{\varepsilon} \cdot \nabla \mathbf{d}_{\varepsilon}\right) \wedge \mathbf{d}_{\varepsilon}-\gamma \nabla \cdot\left(\nabla \mathbf{d}_{\varepsilon} \wedge \mathbf{d}_{\varepsilon}\right)=0 .
$$

Making $\varepsilon \rightarrow 0$, we can deduce (21), using the strong convergences of $\mathbf{u}_{\varepsilon}$ and $\mathbf{d}_{\varepsilon}$ and the weak convergences of $\partial_{t} \mathbf{d}_{\varepsilon}$ and $\nabla \mathbf{d}_{\varepsilon}$. 


\section{Identification of $M=\nabla \mathbf{d} \odot \nabla \mathbf{d}$.}

The key of this proof is to obtain $L^{2}$-compactness for $\nabla \mathbf{d}_{\varepsilon}$, using some ideas of the optimisation framework. Indeed, since $\mathbf{w}_{\varepsilon}=-\Delta \mathbf{d}_{\varepsilon}+\mathbf{f}_{\varepsilon}\left(\mathbf{d}_{\varepsilon}\right)$, then $\mathbf{d}_{\varepsilon}(t)$ can be viewed as a solution of the optimisation (without constraints) problem

$$
J_{\varepsilon}\left(\mathbf{d}_{\varepsilon}(t)\right)=\min _{\mathbf{d} \in H_{\mathbf{h}}^{1}(\Omega)} J_{\varepsilon}(\mathbf{d})\left[=\int_{\Omega}\left(\frac{1}{2}|\nabla \mathbf{d}|^{2}+F_{\varepsilon}(\mathbf{d})-\mathbf{w}_{\varepsilon}(t) \cdot \mathbf{d}\right)\right] .
$$

On the other hand, a.e. $t$ let us define $\tilde{\mathbf{d}}(t)$ as a solution of the optimisation (with constraints) problem

$$
J(\tilde{\mathbf{d}}(t))=\min _{\mathbf{d} \in H_{\mathbf{h}}^{1}(\Omega)|\mathbf{d}|=1} J(\mathbf{d})\left[=\int_{\Omega}\left(\frac{1}{2}|\nabla \mathbf{d}|^{2}-\mathbf{w}(t) \cdot \mathbf{d}\right)\right] .
$$

Obviously, $J_{\varepsilon}\left(\mathbf{d}_{\varepsilon}(t)\right) \leq J_{\varepsilon}(\tilde{\mathbf{d}}(t))$. In particular,

$$
\int_{0}^{T} \int_{\Omega}\left(\frac{1}{2}\left|\nabla \mathbf{d}_{\varepsilon}(t)\right|^{2}+F_{\varepsilon}\left(\mathbf{d}_{\varepsilon}(t)\right)-\mathbf{w}_{\varepsilon}(t) \cdot \mathbf{d}_{\varepsilon}(t)\right) \leq \int_{0}^{T} \int_{\Omega}\left(\frac{1}{2}|\nabla \tilde{\mathbf{d}}(t)|^{2}-\mathbf{w}_{\varepsilon}(t) \cdot \tilde{\mathbf{d}}(t)\right) .
$$

Taking limit inf as $\varepsilon \rightarrow 0$ in (23), bounding previously $F_{\varepsilon}\left(\mathbf{d}_{\varepsilon}(t)\right) \geq 0$,

$$
\begin{aligned}
\int_{0}^{T} J(\mathbf{d}(t)) & \leq \liminf _{\varepsilon \rightarrow 0} \int_{0}^{T} \int_{\Omega}\left(\frac{1}{2}\left|\nabla \mathbf{d}_{\varepsilon}(t)\right|^{2}-\mathbf{w}_{\varepsilon}(t) \cdot \mathbf{d}_{\varepsilon}(t)\right) \\
& \leq \lim _{\varepsilon \rightarrow 0} \int_{0}^{T} \int_{\Omega}\left(\frac{1}{2}|\nabla \tilde{\mathbf{d}}(t)|^{2}-\mathbf{w}_{\varepsilon}(t) \cdot \tilde{\mathbf{d}}(t)\right)=\int_{0}^{T} J(\tilde{\mathbf{d}}(t)),
\end{aligned}
$$

hence one has the equality $\int_{0}^{T} J(\mathbf{d}(t))=\int_{0}^{T} J(\tilde{\mathbf{d}}(t))$ (the opposite inequality is easy to deduce since $|\mathbf{d}(t)|=1)$. Consequently, all the previous inequalities are equalities, and in particular

$$
\exists \lim _{\varepsilon \rightarrow 0} \int_{0}^{T} \int_{\Omega}\left(\frac{1}{2}\left|\nabla \mathbf{d}_{\varepsilon}(t)\right|^{2}-\mathbf{w}_{\varepsilon}(t) \cdot \mathbf{d}_{\varepsilon}(t)\right)=\int_{0}^{T} J(\mathbf{d}(t))=\int_{0}^{T} \int_{\Omega}\left(\frac{1}{2}|\nabla \mathbf{d}(t)|^{2}-\mathbf{w}(t) \cdot \mathbf{d}(t)\right)
$$

Since $\exists \lim _{\varepsilon \rightarrow 0} \int_{0}^{T} \int_{\Omega} \mathbf{w}_{\varepsilon}(t) \cdot \mathbf{d}_{\varepsilon}(t)=\int_{0}^{T} \int_{\Omega} \mathbf{w}(t) \cdot \mathbf{d}(t)$ (using the strong convergence of $\mathbf{d}_{\varepsilon}$ ), one has $\exists \lim _{\varepsilon \rightarrow 0}\left\|\nabla \mathbf{d}_{\varepsilon}\right\|_{L^{2}\left(L^{2}\right)}^{2}=$ $\|\nabla \mathbf{d}\|_{L^{2}\left(L^{2}\right)}^{2}$ therefore,

$$
\mathbf{d}_{\varepsilon} \rightarrow \mathbf{d} \quad \text { in } L^{2}\left(\mathbf{H}^{1}\right) \text {-strong. }
$$

In particular, taking into account the estimates of $\nabla \mathbf{d}_{\varepsilon}$,

$$
\nabla \mathbf{d}_{\varepsilon} \rightarrow \nabla \mathbf{d} \quad \text { in } L^{p}\left(L^{q}\right) \text {-strong, } \forall p, q: p<\infty, q<2,
$$

hence we can identify $M=\nabla \mathbf{d} \odot \nabla \mathbf{d}$.

Acknowledgements. The first author has been partially financed by the projet BFM2000-1317, and the second author by the projects CNPq-Brasil 300116-93-4 and Fapesp-Brasil 01/07557-3 


\section{References}

[1] F. Bethuel, H Brezis, F. HéLein, Asymptotics for the minimization of a Ginzburg-Landau functional, Calc. Var. 1 (1993) 123-148.

[2] Y. Chen, The weak solutions to the evolution problems of harmonic maps, Math. Z. 201 (1989) 69-74.

[3] D. Coutand, S. Shkoller, Well-posedness of the full Ericksen-Leslie model of nematic liquid crystals, Note C.R.A.S., t. 333, Sèrie I (2001) 919-924.

[4] F.H. Lin, Nonlinear theory of defects in nematic liquid crystals: phase transition and flow phenomena, Comm. Pure Appl. Math. 42 (1989) 789-814.

[5] F.H. Lin, C. LiU, Non-parabolic dissipative systems modelling the flow of liquid crystals, Comm. Pure Appl. Math. 48, (1995), 501-537.

[6] F.H. Lin, C. Liu, Existence of solutions for the Ericksen-Leslie system, Arch. Rat. Mech. Anal. 154 (2000) 135-156.

[7] A Prohl, Computational Micro-magnetism, Advances in Numerical Mathematics, Teubner 2001.

[8] S. ShKoller, Well-posedness and global attractors for liquid crystals on Riemannian manifolds, Comm. Partial Differ. Eq. 27, 5 \& 6 (2001) 1103-1137.

[9] J. Simon, Compact sets in $L^{p}(0, T ; B)$, Ann. Mat. Pura Appl., 146 (1987) 65-97.

[10] R. Temam, Navier-Stokes equations, North-Holland Elsevier, 1985.

a Departamento de Ecuaciones Diferenciales y Análisis Numérico, Aptdo. 1160, 41080 Sevilla (Spain). E-mail:guillen@numer.us.es

b Departamento de Matematica Aplicada, IMECC-UNICAMP, C.P. 6065, 13081-970, Campinas-SP (Brasil).

E-mail:marko@ime.unicamp.br 